\title{
CONCEPCIONES SOBRE CIENCIA Y GÉNERO EN EL PROFESORADO DE QUÍMICA: APROXIMACIONES DESDE UN ESTUDIO COLECTIVO DE CASOS
}

\section{Chemistry teachers' conceptions about science and gender: approaches from a collective case study}

\author{
Johanna Patricia Camacho González ${ }^{1}$
}

\begin{abstract}
Resumen: El propósito de esta investigación fue identificar y caracterizar las concepciones del profesorado sobre ciencia y género. Para lograr este objetivo se realizo un estudio de casos colectivo a través de un Curso Taller de Formación Docente en la ciudad de Santiago de Chile. La información recolectada incluyó las respuestas a dos cuestionarios, el discurso del profesorado en los talleres de reflexión docente y la observación directa de la interacción en el aula. El análisis descriptivo interpretativo, permitió identificar perfiles conceptuales de cada caso participante y evidenciar cómo dichos perfiles cambiaban a través del desarrollo de la investigación. Sí bien se encontró que aún persiste una visión androcentrica de la ciencia en el profesorado, al parecer la incorporación de sesiones de reflexión teórica sobre el tema pueden contribuir favorablemente tanto en el cambio de las concepciones como a las práctica pedagógicas.
\end{abstract}

Palabras clave: Ciencia. Género. Estudio de casos. Formación de profesores. Enseñanza de la química.

\begin{abstract}
The objective of this research was to identify and characterize chemistry teachers' conceptions about science and gender. The collective case study was carried out in a Teacher Training Workshop in Santiago-Chile City. The data collected included responses to two questionnaires, the teacher speech in workshops, and direct observation of classroom interaction. The Interpretive descriptive analysis done identifies the conceptual profiles and how these changed through the development of the research. While it was found that there is still a male-centered view of science teachers, incorporating theoretical brainstorming sessions on the subject can apparently contribute positively to conceptual change and educational practice.
\end{abstract}

Keywords: Science. Gender. Case study. Teacher training. Chemistry education.

\footnotetext{
${ }^{1}$ Departamento de Estudios Pedagógicos, Facultad de Filosofía y Humanidades, Universidad de Chile. Avenida Capitán Ignacio Carrera Pinto, 1025, Nuñoa, Santiago, Chile. <jpcamacho@uchile.cl>. Website:

www.johannacamachogonzalez.cl
} 


\section{Introducción}

La relación ciencia, género y educación en la actualidad es considerado como un aspecto fundamental en el desarrollo de la sociedad. Así, la Organización de las Naciones Unidas para la Educación, la Ciencia y la Cultura (UNESCO) desde 1995 lo ha propuesto como objetivo prioritario (UNESCO, 2009; UNESCO, 2010); también la Organización de las Naciones Unidas (ONU, 2000), a través de los objetivos del Desarrollo del Milenio lo ha señalado como el segundo propósito en abordar y la ONU (2009) lo han definido como el tercer objetivo fundamental para el año 2015.

Estas iniciativas pretenden solucionar el problema de desigualdad entre géneros, ya que a pesar de las nuevas perspectivas educativas, políticas y sociales propuestas a nivel mundial y específicamente en Chile, todavía persisten aspectos críticos en particular con la educación de la mujer, a pesar que su rol ha sido realzado en la última década con gran protagonismo en diferentes ámbitos, incluido las ciencias (SALAS, 2006).

Históricamente la ciencia y tecnología se ha caracterizado por un visión tradicional, un contexto dominado principalmente por los hombres, donde las mujeres, sus aportes y necesidades han sido invisibilizados (BUCCHERI; GÜRBER; BRÜHWILER, 2011; SCHIEBINGER, 2004; WATTS, 2007). Diferentes estudios históricos, muestran además que la representación de la mujer ha sido inferior o poco visible en comparación a la imagen de hombres como referente de los aspectos científicos, políticos, económicos, sociales y culturales (BARCLAY et al., 2011); lo que conlleva a suponer una desigualdad de género, en tanto que el rol de la mujer ha sido ignorado en general en la creación de conocimiento científico y el rol masculino, supone la adopción de conceptos y contextos determinados. Estas diferencias según el género cuentan con gran tradición, entendiéndolo

[...] como construcción cultural derivada de la sexuación, categoría cultural impuesta sobre un cuerpo sexuado, facilitaba la distinción entre hechos biológicos y sociales y permitía explicar que las diferencias biológicas entre hombres y mujeres no implicaban, por sí mismas, capacidades, actitudes o aptitudes diferentes. (GARCÍA MESEGUER, 1989 apud BALLARÍN, 2006, p. 12).

De acuerdo con la literatura especializada se considera que la diferencia se debe a procesos biológicos (McCLURE et al., 2004) o que puede ser explicada por los procesos de socialización (UNESCO, 2009).

A continuación, se discuten en profundidad algunos antecedentes que permiten sustentar la importancia que las/los docentes cumplen como un factor determinante en el proceso de socialización (BARBER; MOURSHED, 2007; LABUDDE et al., 2000; TREVIÑO; DONOSO; BONHOMME, 2009), así como el papel que tienen sus concepciones sobre ciencia y género en la promoción de la equidad / desigualdad en el aula de ciencias (FERNÁNDEZ et al., 1995; IZQUIERDO; GARCÍA; SOLSONA, 2009; MANASSERO; VÁSQUEZ, 2003). 
Concepciones sobre Ciencia y género ...

\section{Antecedentes sobre las concepciones de ciencia y género del profesorado}

En cuanto a los factores escolares y en particular al rol del profesorado en los procesos de socialización en el aula, se ha evidenciado que la ausencia en los contenidos escolares de modelos y referentes femeninos (FERNÁNDEZ et al., 1995; IZQUIERDO; GARCÍA; SOLSONA, 2009; PARKER; RENNIE, 2002); las concepciones del profesorado sobre ciencia y género tradicionales (CAMACHO, 2010; LIZANA, 2008; LYNCH; NOWOSENETZ, 2009) y el estilo del profesorado (ORGANISATION FOR ECONOMIC CO-OPERATION AND DEVELOPMENT, 2010; ORGANIZACIÓN PARA COOPERACIÓN Y EL DESARROLLO ECONÓMICO, 2006), afectan principalmente a las jóvenes quienes "encuentran límites, no formales, pero sí reales a sus oportunidades de acceso a determinados estudios" (FERNÁNDEZ et al., 1995, p. 65), antecedentes que dan cuenta de un problema de desigualdad social, en el aula de ciencias.

Desde la investigación en Didáctica de las Ciencias, específicamente relacionada con las concepciones sobre ciencia-género del profesorado, se ha identificado que estas generalmente se caracterizan por ser androcentricas, es decir por situarse sólo desde "una mirada distorsionada y empobrecida de la realidad ya que oculta las relaciones de poder y de posesión del orden simbólico masculino sobre las mujeres [...] un único modelo masculino y un único modelo femenino, enfrentados por oposición, lo que supone una distinta valoración." (GONZÁLEZ; LOMAS, 2006, p. 223).

Según las investigaciones de Camacho (2010), Fernández et al. (1995), Longino (1990), Lynch y Nowosenetz (2009), Manassero y Vásquez (2003), se considera que esta visión androcentrica es sustentada en la concepción más tradicional de la ciencia, cuyas principales características (objetiva, racional, inductiva, neutra y analítica) proyectan una imagen estereotipada masculina y donde, los aspectos relacionados con el contexto valórico, social y cultural (heterogeneidad ontológica, interacción mutua, aplicabilidad a las necesidades) actualmente más promovidos por la UNESCO (2009), son menos reconocidos. Así mismo, se ha encontrado que dichas concepciones conllevan al uso de lenguaje sexista - el uso excesivo del masculino como genérico (BALLARÍN, 2006) -, que se puede distinguir tanto en el discurso del profesorado (CAMACHO, 2010; FERNÁNDEZ et al., 1995), como en los textos escolares (ELGAR, 2004), lo que en algunos casos refuerza una imagen androcentrica y tradicional de la ciencia, independiente de cursos y materias (ELGAR, 2004; MANASSERO; VÁSQUEZ, 2003).

Desde este punto de vista, además, Manassero y Vásquez (2003) señalan que las/los docentes con una concepción tradicional de la ciencia difícilmente aceptará el carácter sexista o la ciencia para todos y todas y cambiará sus prácticas pedagógicas para que dejen de ser excluyentes. Alemany (1992), encontró que en el profesorado existen al menos dos concepciones de las estudiantes "las brillantes" y "las que vienen a conseguir novio" a pesar que sus notas sean mejores que las de los estudiantes, se cree que "ellas no son tan inteligentes". En un estudio del colegio de docentes de Chile, se evidenciaron resultados similares "la buena alumna es principalmente responsable y con menor grado de autonomía y el buen alumno es inteligente y autónomo." (CONTRERAS, 2004, p. 26). Esta representación es sustentada además, por los 
estudios de Alper (1993) y Fernández et al. (1995) con 300 docentes en Inglaterra y España respectivamente, donde tras cambiar el nombre del estudiantado se encontró que la puntuación de las pruebas con nombres de varones era superior a las que tenían nombres de mujeres.

En la investigación de Lizana (2008) además, se develan conclusiones sobre el profesorado de educación básica en formación inicial, las cuales se caracterizan entre otros aspectos, por la clara presencia de estereotipos machistas de la sociedad chilena actual que superponen la incompatibilidad entre la categoría mujer - madre profesional. Lizana (2008) también denota la presencia de patrones machistas en la crianza de los hijos y las hijas lo que orientan a un rol femenino maternal y al posible o eventual abandono de sus actividades profesionales.

Las concepciones del estudiantado generalmente reafirman la visión androcentrica de la ciencia del profesorado (FUNG, 2002). En dos estudios realizados (BRAVO GONZÁLEZ et al., 2009; GONZÁLEZ et al., 2009), encontraron que el estudiantado de educación media tiene una imagen de la persona que se dedica a la actividad científica bajo un estereotipo masculino: hombre, de raza blanca, joven, sonriente, que trabaja solo en un laboratorio, usa con delantal y gafas, cuyas actividades principales están relacionadas con la observación, reflexión y planificación de investigaciones, acciones relacionadas con la visión de ciencia tradicional.

Teniendo en cuenta que las concepciones del profesorado influyen en las concepciones de ciencia-género de los / las estudiantes, se podría pensar sí este proceso de socialización es diferenciado por el género del docente. Según señala el Ministerio de Educación Nacional (CHILE, 2008), tradicionalmente el ejercicio docente ha sido fuertemente dominado por las mujeres $(71 \%)$, la presencia femenina es casi total $(95.5 \%)$ en la educación preescolar, mayoritariamente $(75.5 \%)$ en la educación básica y en la educación media disminuye su proporción (57.5\%). Sin embargo, en hallazgos de diferentes investigaciones se ha evidenciado que el género del profesorado no necesariamente se relaciona con la reproducción de una visión androcentrica, sino que dicho proceso de socialización esta más relacionado con las concepciones del profesorado, como se argumento anteriormente y con la manera en que el/la docente desarrolla su práctica pedagógica, ya que existe evidencia que durante dichas prácticas se reproducen estereotipos de género (IZQUIERDO; GARCÍA; SOLSONA, 2009; LIZANA, 2008; TOMÉ; RAMBLA, 2001).

A partir de los antecedentes discutidos anteriormente, se propone como objetivo de la investigación identificar y caracterizar las concepciones del profesorado de química sobre ciencia y género, a través del desarrollo de un Curso Taller de Historia de la Ciencia y Formación Docente.

\section{Metodología: Diseño Metodológico}

Para abordar dicho objetivo, se optó por el estudio de casos colectivo, una estrategia de diseño de la investigación que según Stake (1999) se define por su interés en casos particulares. El estudio de casos ha sido definido por VanWynsberghe y Khan (2007) como una heurística transparadigmática y transdisciplinar que involucra un cuidadoso delineamiento del fenómeno mediante la evidencia que será recolectada. El estudio de casos se realizó con 2 docentes (Caroline y Emilio) y sus 57 estudiantes, quienes participaron de un Curso Taller sobre Historia de la Química y Formación Docente. Este Curso Taller, tuvo como principal 
característica la promoción de instancias metacognitivas en donde el profesorado pensaba alternativamente como persona que aprende y que enseña. Además, estuvo principalmente enfocado hacia la reflexión y práctica pedagógica para problematizar, reformular esquemas y concepciones que subyacen acerca de la ciencia y su enseñanza (ASTUDILLO; RIVAROSA; ORTIZ, 2008). El Curso Taller, se desarrolló a través de 10 sesiones o Talleres de Reflexión Docente (TRD) de 120 minutos cada uno, estableciendo cuatro fases de investigación, así:

Fase I. Diagnóstico. Durante esta primera los casos estudiados contestaron un cuestionario Tipo Likert (C07) y un cuestionario abierto (C08) sobre las estrategias, ventajas y desventajas de incorporar la Historia de la Ciencia en la actividad química escolar.

Fase II. Fundamentación teórica. Los resultados anteriores conllevaron a proponer el Curso Taller sobre Historia de la Ciencia y Formación Docente, como un espacio de formación y reflexión docente. La fase de fundamentación teórica se desarrollo durante los TRD01-TRD04, en estas sesiones se discutió acerca de las mujeres científicas y su influencia en el desarrollo de la Historia de Ciencia.

Fase III. Diseño didáctico de una unidad didáctica. Esta tercera fase, tuvo como objetivo elaborar un diseño didáctico para la enseñanza de la electroquímica. Para ello, durante las sesiones TRD05-TRD06, se hizo una revisión historiográfica de la Electroquímica entre 1800 a 1853, en el cual se considera el máximo desarrollo histórico según proponen Marcet (1853) y Grapí (2006). En las sesiones TRD07 y TRD08, la discusión y el análisis se centro en el diseño de la Unidad Didáctica Teoría electroquímica en la educación media. Una propuesta didáctica fundamentada en la Historia de la Ciencia en donde se presenta una secuencia didáctica de actividades según el ciclo constructivista de aprendizaje (Sanmartí, 2000).

Fase IV. Implementación y evaluación. Durante esta fase, el profesorado participante implementó la unidad didáctica diseñada a través de 4 sesiones de clase (OBSC1-OBSC4). Además, paralelamente al trabajo desarrollado en el aula, en los talleres finales TRD 09 y TRD 10, se analizo la implementación de la unidad didáctica y algunas producciones de los y las estudiantes.

\section{Plan de análisis}

Una vez se identificaron y seleccionaron las unidades de análisis durante las cuatro fases de la investigación, se procedió a realizar un análisis del contenido del discurso (KRIPPENDORF, 2004). El tratamiento de los datos, se hizo bajo un enfoque cualitativo a través de la propuesta de Miles e Huberman (1994), la cual se caracteriza en la complejidad y no linealidad de las actividades propuestas. Durante la reducción de datos se realizo una codificación abierta de las unidades de análisis seleccionadas y posteriormente, una codificación axial que consistió en relacionar los códigos y a partir de un patrón temático levantar la categoría de análisis Género y Ciencia. A partir de este proceso de reducción, los datos se organizaron a través de matrices, las cuales recibieron el nombre de perfil conceptual, es decir un esquema 
que representa las distintas formas de pensar y hablar que puede tener el profesorado, el cual puede ser utilizado según determinado contexto, como señala Mortimer (1995). La interpretación y conceptualización se realizó según los tipos de contenido (WANG; PETERSEN, 2002), los planos de desarrollo de pensamiento (LABARRERE; QUINTANILLA, 2002), las dimensiones de las competencias de pensamiento científico (QUINTANILLA, 2006) y los aspectos conceptuales desde el racionalismo moderado (IZQUIERDO, 2005; TOULMIN, 1977), según como se propone en el Cuadro 1. Este análisis permitió una interpretación conceptual desde diferentes niveles relacionados entre sí para dar cuenta sobre cuál es el perfil conceptual del profesorado y cómo este perfil cambia en una u otro nivel a través del proceso de intervención. Finalmente, se realizó una triangulación metodológica por métodos.

\section{Resultados}

\section{Resultados y análisis del estudio de caso Caroline}

Fase I. Diagnóstico. Con respecto a las concepciones de la profesora Caroline, ella manifiesta que "la relación entre los problemas cientificos de la época con los requerimientos del hombre" (C081.3), así a través de esta afirmación además se evidencia una "imagen masculinizada de ciencia" (C081.3) que se contrasta con otra afirmación que hace en el mismo momento (C08) relacionando la rigurosidad metodológica con características masculinas, "en relación a la metodología cientifica y la rigurosidad en el trabajo cientifico, asi como a la necesidad de esto como requerimiento del hombre de ciencia”. (C081.2)

Fase II. Fundamentación teórica y Fase III. Diseño didáctico de una unidad Didáctica. Durante estas fases, el perfil conceptual de Caroline sobre Ciencia y Género, se caracteriza por reconocer principalmente el valor de las mujeres en la actividad científica desde una perspectiva conceptual, relacionada con la "producción de las mujeres en el desarrollo de diferentes conceptos químicos" (TRD1.079), así como "el valor de los experimentos" (TRD1.079) a pesar de considerar que "las mujeres no tenían interés por el trabajo experimental o no se situaban en los laboratorios de ciencia” (TRD1.083) y "que quizás sus contribuciones las realizaban a la escondida” (TRD1.080). Además, señala el valor conceptual (O1) del aporte de las mujeres en las ciencias y lo reconoce desde un plano social (P3) que permiten articular dicho aspecto con la representación general de los objetos o fenómenos estudiados (R1) con los contenidos conceptuales (Q1).

Fase IV. Implementación y evaluación. Acerca del género en ciencias se destacan tres aspectos importantes durante esta última fase. El primer consiste en la continuidad de la imagen de mujeres como reprimidas socialmente, concepción que es compartida por sus estudiantes, quienes consideran "que ellas no tenian libertad de expresión o que no eran tomadas en cuenta" (OBSC1.015; OBSC1.017; OBSC1.018; OBSC1.020). La profesora Caroline valora el aporte de las mujeres en ciencias e identifica la relevancia del trabajo femenino en la actividad química, sin embargo aún prevalece la idea de este aporte "en esa época el trabajo científico era más bien relacionado con el hombre" (OBSC1.021), ya que "los temas están más relacionados con los apellidos de hombres y no se les da la importancia” (OBSC1.021). Identifican mujeres desde una visión 'auxiliar' 
Concepciones sobre Ciencia y género ...

Cuadro 1. Matriz de análisis de componentes para la interpretación conceptual de las categorías propuestas.

\begin{tabular}{|c|c|c|c|}
\hline \multirow{3}{*}{$\begin{array}{l}\text { Tipo de } \\
\text { contenido } \\
\text { (WANG; } \\
\text { PETERSEN, } \\
\text { 2002) }\end{array}$} & Conceptual & Procedimental o metodológico & Contextual \\
\hline & 01 & $\mathrm{O} 2$ & O3 \\
\hline & $\begin{array}{l}\text { Problematización y relación de } \\
\text { los contenidos con la naturaleza } \\
\text { del conocimiento. Énfasis en la } \\
\text { naturaleza tentativa del } \\
\text { conocimiento científico }\end{array}$ & $\begin{array}{l}\text { Procesos y diseño de } \\
\text { experimentosLas dinámicas de } \\
\text { las comunidades científicas } \\
\text { (Métodos de investigación) }\end{array}$ & $\begin{array}{l}\text { La actividad química en } \\
\text { relación con los aspectos } \\
\text { individuales y colectivos } \\
\text { Factores socio-culturales }\end{array}$ \\
\hline \multirow[b]{3}{*}{$\begin{array}{l}\text { Planos de } \\
\text { Desarrollo del } \\
\text { Pensamiento } \\
\text { (LABARRERE; } \\
\text { QUINTANILLA, } \\
\text { 2002) }\end{array}$} & Personal - Significativo & Instrumental operativo & Social o comunicativo \\
\hline & P1 & P2 & P3 \\
\hline & $\begin{array}{l}\text { Los procesos y estados } \\
\text { personales de quien resuelve el } \\
\text { problema resultan ser } \\
\text { relevantes y la atención del } \\
\text { sujeto deja a un lado el análisis } \\
\text { de la situación, la búsqueda } \\
\text { activa de instrumentos, las } \\
\text { representaciones de finalidades } \\
\text { vinculadas con la solución } \\
\text { esperada se centra en la } \\
\text { persona como sujeto de la } \\
\text { solución. }\end{array}$ & $\begin{array}{l}\text { Identifica aquellos momentos o } \\
\text { fragmentos del enfrentamiento y } \\
\text { solución de los problemas en que } \\
\text { los recursos del sujeto o del } \\
\text { grupo que los resuelve están } \\
\text { centrados en aspectos tales como } \\
\text { el contenido, las relaciones que } \\
\text { los caracterizan, las soluciones } \\
\text { posibles y las estrategias, } \\
\text { procedimientos y así por el estilo. }\end{array}$ & $\begin{array}{l}\text { Espacio generado en la } \\
\text { solución grupal de problemas } \\
\text { o en la interacción netamente } \\
\text { pedagógica, hace referencia } \\
\text { no sólo a las relaciones que } \\
\text { constituye la trama que se } \\
\text { teje en los procesos } \\
\text { comunicativos de los } \\
\text { alumnos, sino también al } \\
\text { conocimiento y la } \\
\text { representación que los } \\
\text { sujetos tienen de esas } \\
\text { interacciones }\end{array}$ \\
\hline \multirow{3}{*}{$\begin{array}{l}\text { Competencias } \\
\text { de } \\
\text { Pensamiento } \\
\text { Científico } \\
\text { (QUINTANILLA, } \\
\text { 2006) }\end{array}$} & Saber conocer & Saber hacer & Saber ser \\
\hline & Q1 & Q2 & Q3 \\
\hline & $\begin{array}{l}\text { Involucra el manejo de las } \\
\text { estrategias para ser } \\
\text { conscientes y para regular el } \\
\text { proceso de aprendizaje } \\
\text { personal. }\end{array}$ & $\begin{array}{l}\text { Es el saber de la actuación en la } \\
\text { realidad, de forma sistemática y } \\
\text { reflexiva, buscando la } \\
\text { consecución de metas, de } \\
\text { acuerdo con determinados } \\
\text { criterios. }\end{array}$ & $\begin{array}{l}\text { Consiste en la articulación } \\
\text { de valores, actitudes y } \\
\text { normas enmarcadas en el } \\
\text { desempeño por } \\
\text { competencias. }\end{array}$ \\
\hline \multirow{3}{*}{$\begin{array}{l}\text { Racionalismo } \\
\text { Moderado } \\
\text { (TOULMIN, } \\
\text { 1977; } \\
\text { IZQUIERDO, } \\
\text { 2005) }\end{array}$} & Técnicas de representación & Procedimientos de aplicación & Lenguaje \\
\hline & R1 & R2 & R3 \\
\hline & $\begin{array}{l}\text { Las relaciones generales } \\
\text { discernibles entre objetos, } \\
\text { sucesos y fenómenos } \\
\text { Modelo teórico al cual } \\
\text { pertenecen }\end{array}$ & $\begin{array}{l}\text { Supone también la aptitud para } \\
\text { someter aprueba y delinear las } \\
\text { fronteras del <<ámbito>>0 } \\
<<\text { rango de aplicación>>, dentro } \\
\text { del cual esos símbolos y técnicas } \\
\text { de representación tienen genuina } \\
\text { relevancia empírica } \\
\text { Operaciones que se pueden } \\
\text { realizar }\end{array}$ & $\begin{array}{l}\text { Incluye los términos técnicos } \\
\text { o nombres de conceptos y } \\
\text { también las oraciones, sean } \\
\text { leyes naturales o } \\
\text { generalizaciones directas. } \\
\text { Símbolos, representaciones } \\
\text { gráficas, ecuaciones básicas }\end{array}$ \\
\hline
\end{tabular}

Fuente: elaborado por la autora. 
la "esposa de un científico" (OBSC1.032). Además, se identifica un nivel conceptual del género; en particular con el plano de desarrollo social o contextual, al mencionar el valor del trabajo de las mujeres en la actividad química. Así, el perfil conceptual de Caroline muestra que la visión de género en ciencias está relacionado desde un saber ser (Q3) que relaciona el lenguaje (R3) desde un contenido procedimental (O2) y un plano social comunicativo (P2).

Con respecto a las concepciones de Caroline acerca de la Ciencia y género se aprecia que su perfil conceptual (Cuadro 2) presenta transiciones entre uno y otro aspecto a lo largo de las distintas fases de investigación. En particular con respecto al conocimiento científico se observó que durante la fase de diagnóstico sus concepciones estaban más orientadas desde un plano personal significativo (P1) y que existió un cambio hacia el plano social comunicativo (P3) el cual se mantuvo hasta la última fase de implementación y evaluación. Acerca del contenido también se evidenció un cambio del contenido conceptual (O1) hacia el contenido contextual (O1), este cambio se evidenció durante la última fase de investigación donde se desarrollo la unidad didáctica con sus estudiantes. Con respecto a la dimensión de la competencia, se identifican varias transiciones desde el saber conocer (Q1), pasando por el saber hacer (Q2) que se evidenció con fuerza durante las fases II y III, hasta el saber ser y comunicar (Q3) cerrando la investigación, lo que tiene coherencia con la transición entre los planos de conocimiento. Por último, se observó acerca del racionalismo moderado de la actividad científica que al inicio estuvo en relación de los modelos teóricos (R1), pasando por las técnicas de aplicación, durante las fases II y III, hacia la importancia del lenguaje en la construcción de conocimiento científico.

Cuadro 2. Perfil conceptual del estudio de caso Caroline sobre ciencia y género.

\begin{tabular}{|c|c|c|c|c|c|c|c|c|c|c|c|c|c|c|c|}
\hline \multicolumn{10}{|c|}{ Perfil conceptual del estudio de caso Caroline sobre género y ciencia } \\
\hline O Diagnóstico & \multicolumn{3}{c|}{ Dundamentación } & \multicolumn{3}{c|}{$\begin{array}{c}\text { Diseño } \\
\text { evaluación }\end{array}$} \\
\hline 3 & 3 & 1 & 1 & 1 & 3 & 1 & 1 & 1 & 3 & 1 & 1 & 2 & 3 & 1 & 1 \\
\hline
\end{tabular}

Contenido Conceptual O1, Contenido Procedimental O2, Contenido Contextual O3;

Plano Personal P1, Plano Instrumental P2, Plano Social P3;

Saber Conocer Q1, Saber Hacer Q2, Saber Ser Q3;

Representación R1, Experiencia R2, Lenguaje R3.

Fuente: elaborado por la autora.

Así, las concepciones de la profesora Caroline tenían un claro énfasis en la promoción de los modelos teóricos y el plano personal significativo, sin embargo durante el modelo de intervención sus concepciones se vieron orientadas más hacia el plano de desarrollo social y comunicativo, allí otorgó importancia no sólo a los saberes conceptuales como se identificaba en el principio, sino que también a los aspectos contextuales de la actividad científica. A pesar de estos cambios importantes en las concepciones de Caroline también es importante señalar que prevalecieron algunas concepciones androcentricas, sí bien se reconocen las mujeres científicas como un aspecto importante en la educación química, esto sólo se evidenció para una comprensión contextual en la enseñanza de la electroquímica (Cuadro 2). 


\section{Resultados y análisis del estudio de caso Emilio}

Fase I. Diagnóstico. Con respecto a las concepciones de Emilio sobre ciencia y género se observó su relación con contenidos contextuales (O3) desde un plano social (P3), "Valorarian las contribuciones de los cientificos a las necesidades del hombre" (C082.02).

Fase II. Fundamentación teórica. El género en ciencias durante la fase II es muy complejo de interpretar, ya que se evidencian concepciones relacionadas principalmente con el plano social y el contenido contextual. Él valora mucho el saber hacer de las mujeres y lo relaciona principalmente con la representación y el lenguaje, siendo caracterizado por una fuerte visión androcentrica de la ciencia. Sí bien reflexiona, cuestiona y analiza el papel de la mujer durante la construcción de conocimiento científico, a través de su discurso presenta una clara visión androcentrica de la ciencia, como se evidencia a continuación: "esta caballero hizo tal cosa" (TRD2.010); "lo que hace el científico" (TRD2.020; TRD2.028), "para ir enseñando la ley del cientifico" (TRD2.029)", entre otras, además, se perciben algunas ideas sobre la superioridad del hombre respecto a la mujer y la necesidad de ella por alcanzar la perfección del trabajo científico, como lo hace el hombre. Por ejemplo en las siguientes afirmaciones "sí las mujeres fueran preparadas en eso, lo podrían hacer, perfecta, tal como lo hace el hombre" (TRD2.064); "que sí se le enseñará a la mujer acerca, todo lo que ella estaba entregando, podrian hacerlo perfectamente bien y trabajar igual que los hombres" (TRD2.065). Con respecto a las mujeres en ciencias él distingue las "mujeres cientificas en la antigüedad de las mujeres cientificas actuales" (TRD2.069) y las caracteriza desde perfiles antagónicos. Al respecto, expresa su valor por las mujeres en la antigüedad, las que considera "era bien bumildes" (TRD2.069); "analiticas" (TRD2.063), "se dedicaban a la familia a los hijos" (TRD2.074), y expresa su valoración por el trabajo de ellas porque considera que estaban relacionadas con diferentes ámbitos sociales como "la salud" (TRD2.053), "los cosméticos" (TRD2.054), a pesar de considerar "que se mantenían ocultas" (TRD2.060) y que la tradición de conocimientos científicos se realizaba por la "verbalización ... no sabian escribir, pero que bablaban todo lo que quisieran" (TRD2.073). Sin embargo, con respecto a las mujeres científicas actuales señala que "la mujer de esta época es diferente a la de ahora, ahora es competitiva" (TRD2.070); "ahora quiere ganar más que el hombre" (TRD2.071); "busca cada vez un mejor estatus" (TRD2.083); "muy de vanidad, mucha obsesión" (TRD2.085); "se descuidan de los aspectos humanos" (TRD2.086). Esta visión se observó con gran fuerza en el discurso, incluso al referirse a las otras profesoras durante el taller de reflexión docente hacía alusión a ellas como mujeres científicas actuales, "hoy es el día de las científicas...te elevo un poquito más el ego por tener una vocal más” (profesor A) (TRD2.089).

Fase III. Diseño didáctico de una unidad didáctica. Al igual que en la fases anteriores Emilio, sólo se refiere a las personas desde una perspectiva masculina habla de ellos, los alumnos y a pesar que denota preocupación en incorporar a las demás personas, como se evidencio por ejemplo al hablar de sus estudiantes"entonces hay un problema abi de poco reconocimiento del aporte que pueden dar la otra persona" (TRD2.131), la visión androcentrica persiste. Esta noción además la continúa relacionando con los contenidos conceptuales (O1), el plano social (P3), el lenguaje (R3) "ellos están realizando una buena explicación, entonces se trata de que lo crean el texto” (TRD2.147) y el saber hacer (Q2) “ellos van a estar observando, ellos respondan” (TRD2.138). 
Fase IV. Implementación y evaluación. En cuanto a la ciencia y género, en esta última fase, se observó que el cambio sólo se realizó acerca del contenido existiendo una transición desde lo conceptual (Q1) hacia lo contextual (O3) y el plano social (P3), teniendo en cuenta que esta dimensión es importante para la construcción de modelos teóricos (R1).

Acerca de las concepciones del profesor Emilio, se identifica inicialmente una fuerte tendencia hacia una visión más dogmática relacionada con el carácter experimental de la química. Fue constante que sus concepciones tuvieran un afán por el saber hacer, por los contenidos procedimentales o por las técnicas de aplicación. En general estás concepciones estaban muy asociadas desde el plano instrumental procedimental, no obstante se evidenció también la importancia que otorga a la enseñanza de los modelos teóricos de la química, que para el se caracterizan en sistemas rigurosos, validos y objetivos, características más relacionadas con la visión de ciencia androcentrica (Cuadro 3).

Cuadro 3. Perfil conceptual del estudio de caso Emilio sobre género y ciencia

\begin{tabular}{|c|c|c|c|c|c|c|c|c|c|c|c|c|c|c|c|}
\hline \multicolumn{10}{|c|}{ Perfil conceptual del estudio de caso Emilio sobre naturaleza de la ciencia } \\
\hline O & P & Q & R & O & P & Q & R & O & P & Q & R & O & P & Q & R \\
\hline 3 & 3 & 1 & 1 & 3 & 3 & 1 & 1 & 1 & 3 & 2 & 3 & 3 & 3 & 2 & 1 \\
\hline
\end{tabular}

Contenido Conceptual O1, Contenido Procedimental O2, Contenido Contextual O3;

Plano Personal P1, Plano Instrumental P2, Plano Social P3;

Saber Conocer Q1, Saber Hacer Q2, Saber Ser Q3;

Representación R1, Experiencia R2, Lenguaje R3;

Fuente: elaborado por la autora.

En cuanto a las concepciones sobre género y ciencias, sólo se identificó un cambio con respecto al contenido, es decir que se pasa de un contenido contextual (O3) en el que se abordaba la mujeres en ciencias para dar contexto, durante la fase de diagnóstico, hacia un contenido más conceptual (O1) que se distingue fuertemente durante las fases de fundamentación teórica y diseño, hacia uno contextual nuevamente (O3), que es la concepción que refleja al implementar las clases de teoría electroquímica con sus estudiantes. En cuanto al plano se mantiene durante toda la investigación desde lo social comunicativo (P3), así como la importancia de saber conocer (Q1) sobre las mujeres científicas para la construcción de modelos teóricos (R1).

Así Emilio presenta en su perfil conceptual (Cuadro 3), varias alusiones en relación al carácter instrumental, al parecer el carácter experimental de las ciencias es importante para la construcción de modelos teóricos y esto puede estar relacionado con aspectos más contextuales como con el género en ciencias y el valor de las personas que construyen el conocimiento científico. 


\section{Resultados y análisis sobre el estudio de casos colectivo}

En el estudio de casos se identificaban que las concepciones sobre ciencia y género, fueron transitando de un plano a otro, señalando así la complejidad del cambio conceptual. Estos cambios se evidenciaron de manera más clara, para ambos casos, en la fase de fundamentación teórica y en la de implementación - evaluación, al parecer generar espacios de reflexión, discusión e intercambio de nociones teóricas permite promover espacios metacognitivos donde el profesorado es capaz de identificar sus propias concepciones, orientarlas hacia nuevos propósitos y ponerlas en juego durante su práctica. La fase de diseño de la Unidad Didáctica fue trabajaba desde una perspectiva más instrumental, estaba el interés por saber cómo trabajarían con sus estudiantes y para esto, retomaron sus maneras tradicionales de hacerlo, llevando la discusión hacia lo que el estudiante debería hacer o no y cómo lo debería realizar, teniendo en cuenta también que la teoría electroquímica, es un tema curricular que habían abordado todos los años de una manera instrumental y por tanto, trabajar con nuevos propósitos y resignificar su enseñanza fue un proceso complejo, que le constó y tal vez por ello, se explica sus concepciones en relación a los aspectos procedimentales durante esta fase.

\section{Conclusiones}

En el profesorado de química participante de la investigación se evidenció que sus concepciones acerca de ciencia y género, se sitúan desde diferentes perspectivas teóricas, es decir coexisten las visiones tradicional- dogmática y la constructivista en general, estos resultados están en concordancia con otras investigaciones en el campo de la Didáctica de las Ciencias Experimentales.

Sin embargo, se hace evidente que las fases de fundamentación teórica y diseño didáctico, permiten además de comprender la construcción de conocimiento científico desde una perspectiva social, relacionar personas con la construcción de sus propios conocimientos científicos, desplazando dichas concepciones de un plano instrumental hacia un plano personal significativo o social comunicativo, esto se manifiesta con mayor fuerza durante la fase de evaluación e implementación, en particular cuando el profesorado desarrolla la clase de electroquímica con sus estudiantes. Estas conclusiones son coherentes con lo que la literatura especializada en este campo nos orienta en torno a la importancia de la problematización del pensamiento y práctica del profesorado como principal característica de los modelos de intervención (ASTUDILLO; RIVAROSA; ORTIZ, 2008; COPELLO; SANMARTÍ, 2001; MELLADO, 2001;).

No obstante, se sitúa como aspecto problemático la noción de cómo ocurre esta construcción del conocimiento científico, sí bien la ciencia es una actividad humana al parecer la elaboración de conocimiento científico en la escuela, se caracteriza por procesos lineales, acumulativos, rigurosos, sistemáticos, que le otorgan mayor validez a la actividad científica. El método científico de la tradición baconiana aún se sitúa en el discurso del profesorado, con algunos matices, donde características como la observación, la sistematización, la objetividad de los datos, la rigurosidad en la experimentación permiten un resultado correcto e incuestionable de la actividad científica, lo que conlleva a concluir que las concepciones sobre ciencia- 
género en el profesorado de ciencias en ejercicio generalmente son tradicionales, es decir principalmente corresponde a una visión androcentrica.

Además, quedó en evidencia que los casos estudiados conocen poco sobre las mujeres científicas y sus aportes a la química, lo que ha sido señalado desde la literatura especializada (ÁLVAREZ; 2006; SOLSONA, 2006). Se reconocen sólo algunos casos célebres de mujeres científicas de la antigüedad, como María la Judía y Marie Curie, además que las caracterizan como esposas, colegas, reprimidas, ocultas, razones por la cual hoy se les reconocen sus aportes. A diferencia del amplio y reconocido rol exitoso del hombre de ciencia, el sabio, el científico, en relación a la producción y acumulación de conocimiento científico, se otorga mayor valor al trabajo científico proveniente de un hombre, además por ser el más conocido. Y un rol rezagado a la mujer, quien se dedicaba a la ciencia a través de la retórica principalmente por cuestiones más sociales o tradicionales como la salud, la producción de cosméticos, aspectos culinarios, la transmisión de conocimientos a nuevas generaciones, etc., estos aspectos fueron particularmente enfatizados durante la enseñanza de la electroquímica en las clases analizadas.

En particular en el profesor Emilio, se denotó que sus concepciones suponían un antagonismo entre los roles de mujer y científica, sobre todo en el contexto actual, él consideraba que la mujer científica de hoy tiene características que rompen su rol tradicional, es ambiciosa, competitiva y descuida su familia, lo que supone un antagonismo entre el rol de madre y científica, aspectos que también se han identificado en el profesorado de Pedagogía en Educación Básica, como lo señalan los resultados de Lizana (2008).

Las anteriores conclusiones merecen principal atención y proponen nuevos desafíos para la educación científica principalmente en algunos países de América como Colombia, Estados Unidos, Chile, y México en donde según los resultados de PISA 2009 aún se identifican diferencias significativas que afectan principalmente a las jóvenes (ORGANISATION FOR ECONOMIC CO-OPERATION AND DEVELOPMENT, 2010). En acuerdo con lo ya señalado por Treviño, Donoso y Bonhomme (2009), es necesario trabajar la visión de género en ciencias con el profesorado, ya que como señalan Manassero y Vásquez (2003) estás resultan ser determinantes para la acción de los estereotipos de género en el aula y muchas veces de manera inconsciente a través del discurso y quehacer profesional, las concepciones del profesorado influyen en la motivación y los intereses del estudiantado, así como en la percepción que puede tener el estudiantado con respecto a la aplicabilidad de las ciencias en la vida cotidiana (BRICKHOUSE; LOWERY; SCHULTZ, 2000). Por ello, se sugiere proponer nuevas estrategias de fundamentación teórica e implementación en el aula que contribuyan a superar la visión androcentrica de la ciencia y así, se otorgue un valor menos ingenuo sobre la participación de las mujeres en la química.

\section{Agradecimientos}

La autora agradece el apoyo del Proyecto FONDECYT 11121249. 
Concepciones sobre Ciencia y género $\ldots$

\section{Referencias}

ALEMANY, C. Yo también he jugado con Electro-L (alumnas en enseñanza superior técnica). Madrid: Instituto de la Mujer, 1992.

ALPER, J. The pipeline is leaking women all the way along. Science, Washington, v. 260, p. 409-411, 16 Apr. 1993.

ÁLVAREZ, M. La historia de la ciencia en la formación del profesorado de ciencias naturales. In: QUINTANILLA, M.; ADÚRIZ-BRAVO, A. (Ed.). Enseñar ciencias en el nuevo milenio: retos y desafíos. Santiago: Pontificia Universidad Católica de Chile, 2006. p. 239-256.

ASTUDILLO, C.; RIVAROSA, A.; ORTIZ, F. El discurso en la formación de docentes de ciencias: un modelo de intervención. Revista Iberoamericana de Educación, Madrid, v. 45 , n. 4 , p. $1-14,2008$.

BALLARÍN, P. Historia de la coeducación. In: GUÍA de buenas prácticas para favorecer la igualdad entre hombres y mujeres en educación. [S.1.]: Consejería de Educación: Junta de Andalucía, 2006. p. 8-17.

BARBER, M.; MOURSHED, M. How the world's best-performing school systems come out on top. [S.1.: s.n.], 2007. Disponible en: <http://www.todospelaeducacao.org.br/ biblioteca/1109/how-the-worlds-best-performing-school-systems-come-out-on-top $>$. Visitado en: 10 jun. 2011.

BARCLAY, K. et al. Gender and generations: women and life cycles. Women's History Review, Abingdon, v. 20, n. 2, p. 175-188, 2011.

BRAVO GONZÁLEZ, P. et al.¿Qué está haciendo el científico?: análisis de la actividad científica descrita por alumnos secundarios chilenos de $11^{\circ}$ y $12^{\circ}$ grado de distintos tipos de establecimientos educacionales. Enseñanza de las Ciencias, Barcelona, p. 2955-2958, 2009. (Número extra).

BRICKHOUSE, N.; LOWERY, P.; SCHULTZ, K. What kind of girls does science?: the construction of school science identities. Journal of Research in Science Teaching, New York, v. 37, n. 5, p. 441-458, 2000.

BUCCHERI, G.; GÜRBER, N. A.; BRÜHWILER, C. The impact of gender on interest in science topics and the choice of scientific and technical vocations. International Journal of Science Education, London, v. 33, n. 1, p.159-178, 2011.

CAMACHO, J. P. Concepciones del profesorado y promoción de la explicación científica en la actividad química escolar: aportes de un modelo de intervención desde la historia de la ciencia para la enseñanza de la electroquímica. 2010. 301 p. Tesis (Doctorado en Ciencias de la Educación) - Facultad de Educación, Pontificia Universidad Católica de Chile, Santiago, 2010.

CHILE. Ministerio de Educación Nacional. Anuario estadístico. Santiago, Chile, 2008. 
Camacho González, J. P.

CONTRERAS, A. Educación y género: un desafío pendiente a la organización magisterial. Santiago, Chile: SERNAM, 2004.

COPELLO, M. I.; SANMARTÍ, N. Fundamentos de un modelo de formación permanente del profesorado de ciencias centrado en la reflexión dialógica sobre las concepciones y las prácticas. Enseñanza de las Ciencias, Barcelona, v.19, n. 2, p. 269-283, 2001.

ELGAR, A. G. Science textbooks for lower secondary schools in Brunei: issues of gender equity. International Journal of Science Education, London, v. 26, n. 7, p. 875-894, 2004.

FERNÁNDEZ, C. et al. Una mirada no sexista a la clase de ciencias experimentales. Barcelona: Institut de Ciénces de 1'Educación, 1995.

FUNG, Y. Y. H. A comparative study of primary and secondary school students' images of scientists. Research in Science \& Technological Education, Abington, v. 20, n. 2, p. 199-213, 2002.

GONZÁLEZ, A.; LOMAS, C. Mujer y educación: educar para la igualdad, educar desde la diferencia. Barcelona: Grao, 2006.

GONZÁLEZ, C. et al. Científicos jóvenes y sonrientes: la imagen de científico de los estudiantes chilenos de $11^{\circ}$ y $12^{\circ}$ grado en diferentes contextos escolares. Enseñanza de las Ciencias, Barcelona, p. 2548-2552, 2009. (Número extra).

GRAPÍ, P. L'electrificació de la química al segle XVIII: una xarxa de guspires. Actes d'Història de la Ciència i de la Tècnica, nova època, Barcelona, v. 1, n. 1, p. 65-74, 2006.

IZQUIERDO, M. Com fer problemàtiques els problemes que no en són prou: noves temàtiques per als problemes de química. In: IZQUIERDO, M. (Ed). Resoldre problemes per aprendre. Bellatera: Universitat Autònoma de Barcelona, 2005. p. 45-52.

IZQUIERDO, M.; GARCÍA, C.; SOLSONA, N. Géner i ensenyament de les ciénces: representaciones i propostes. Bellatera: Universitat Autònoma de Barcelona, 2009.

KRIPPENDORF, K. Content analysis: an introduction to its methodology. Thousand Oaks: Sage, 2004.

LABARRERE, A.; QUINTANILLA, M. La solución de problemas científicos en el aula: reflexiones desde los planos de análisis y desarrollo. Pensamiento Educativo, Santiago, v. 30, n. 1, p. 121-137, 2002. Disponible en: <http://pensamientoeducativo.uc.cl/index.php/pel/ article/view/216 >. Visitado en: 26 mar. 2013.

LABUDDE, P. et al. Girls and physics: teaching and learning strategies tested by classroom interventions in grade 11. International Journal of Science Education, London, v. 22, n. 2, p. 143-157, 2000.

LIZANA, V. A. Representaciones sociales sobre feminidad de los / las estudiantes de pedagogía, en los contextos de formación docente inicial. Estudios Pedagógicos, Valdivia, v. 34, n. 1, p. 115-136, 2008. 
LONGINO, H. E. Science as social knowledge: values and objectivity in scientific inquiry. Pricenton: Princenton University Press, 1990.

LYNCH, I.; NOWOSENETZ, T. An exploratory study of students' constructions of gender in science, engineering and technology. Gender and Education, Abingdon, v. 21, n. 5 , p. 567- 581, 2009.

MANASSERO, M. A.; VÁSQUEZ, A. Los estudios de género y la enseñanza de las ciencias. Revista de Educación, Madrid, n. 330, p. 251-280, 2003.

MARCET, J. H. Conversations on chemistry: in which the elements of that science are familiarly explained and illustrated by experiments. London: Longman, Brown, Green \& Longman, 1853. v. 1.

McCLURE, E. B. et al. A developmental examination of gender differences in brain engagement during evaluation of threat. Biological Psychiatry, Amsterdam, v. 55, n. 11, p. 1047-1055, 2004.

MELLADO, V. ¿Por qué a los profesores de ciencias nos cuesta tanto cambiar nuestras concepciones y modelos didácticos? Revista Interuniversitaria de Formación del Profesorado, Zaragoza, n. 40, p. 17-30, 2001.

MILES, M. B.; HUBERMAN, A. M. An expanded sourcebook: qualitative data analysis. Thousand Oaks: Sages, 1994.

MORTIMER, E. F. Conceptual change or conceptual profile change? Science $\boldsymbol{\&}$ Education, Dordrecht, v. 4, n. 3, p. 267-285, 1995.

ONU. Goals, targets and indicators. [S.1.], 2000. Disponible en: <http:// www.unmillenniumproject.org/goals/gti.htm\#goal3>. Visitado en: 23 jun. 2011.

ONU. The milennium development goals report 2009. New York, 2009. Disponible en: $<$ http://www.un.org/millenniumgoals/pdf/MDG_Report_2009_ENG.pdf > . Visitado en: 10 jun. 2011.

ORGANISATION FOR ECONOMIC CO-OPERATION AND DEVELOPMENT. PISA 2009 results: what students know and can do: student performance in reading, mathematics and science. Paris: OECD, 2010. v. 1. Disponible en: <http://dx.doi.org/ 10.1787/9789264091450-en> . Visitado en: 23 jun. 2011.

ORGANIZACIÓN PARA COOPERACIÓN Y EL DESARROLLO ECONÓMICO. PISA 2006: marco de la evaluación. Conocimientos y habilidades en Ciencias, Matemática y Lectura. París, OECD, 2006.

PARKER, L. H.; RENNIE, L. J. Teachers' implementation of gender-inclusive instructional strategies in single-sex and mixed-sex science classrooms. International Journal of Science Education, London, v. 24, n. 9, p. 881-897, 2002. 
QUINTANILLA, M. Identificación, caracterización y evaluación de competencias de pensamiento científico desde una imagen naturalizada de la ciencia. In: QUINTANILLA, M.; ADÚRIZ-BRAVO, A. (Ed.). Enseñar ciencias en el nuevo milenio: retos y desafíos. Santiago: Pontificia Universidad Católica de Chile, 2006. p. 18-42.

SALAS, E. Las mujeres chilenas que recibieron el siglo $\mathbf{X X}$ y las que lo despidieron. Santiago, Chile: Productora Gráfica Andros, 2006.

SANMARTÍ, N. El diseño de unidades didácticas. In: PERALES PALACIOS, F. J.; CAÑAL DE LEÓN, P. (Coord.). Didáctica de las ciencias experimentales: teoría y práctica de la enseñanza de las ciencias. Madrid: Marfil, 2000. p. 239- 266.

SCHIEBINGER, L. ¿Tiene sexo la mente? Valencia: Cátedra, 2004.

SOLSONA, N. Las mujeres en la historia de la ciencia. In: QUINTANILLA, M.; ADÚRIZBRAVO, A. (Ed.). Enseñar ciencias en el nuevo milenio: retos y desafíos. Santiago: Pontificia Universidad Católica de Chile, 2006. p. 37-63.

STAKE, R. E. Investigación con estudio de casos. Madrid: Morata, 1999.

TOMÉ, A.; RAMBLA, X. Contra el sexismo: coeducación y democracia en la escuela. Madrid: Síntesis, 2001.

TOULMIN, S. E. La comprensión humana. Madrid: Alianza, 1977.

TREVIÑO, E.; DONOSO, F.; BONHOMME, M. ¿Cómo las escuelas chilenas pueden mejorar el aprendizaje en ciencias? In: CARIOLA, L. et al. (Coord.). ¿Qué nos dice PISA sobre la educación de los jóvenes en Chile?: nuevos análisis y perspectivas sobre los resultados en PISA 2006. Santiago, Chile: Ministerio de Educación, 2009. p. 71-104.

UNESCO. Aportes para la enseñanza de las ciencias del SERCE. Santiago, Chile, 2009.

UNESCO. Sex-disaggregated data: a brief analysis of key education and science indicators since the Beijing Declaration and Platform for Action. Montreal: UNESCO Institute for Statistics, 2010. Disponible en: <http://unesdoc.unesco.org/images/0021/ 002171/217139e.pdf>. Visitado en: 10 jun. 2011.

VANWYNSBERGHE, R.; KHAN, S. Redefining case study. International Journal of Qualitative Methods, Edmonton, v. 6, n. 2, p. 80-94, 2007.

WANG, H.; PETERSEN, D. A comparison of elementary, secondary and student teachers' perceptions and practices related to history of science instruction. Science $\boldsymbol{\&}$ Education, Amsterdam, v. 11, n. 1, p. 69-81, 2002.

WATTS, R. Whose knowledge?: gender, education, science and history. History of Education, Abingdon, v. 36, n. 3, p. 283-302, 2007.

Artigo recebido em 23/08/12. Aceito em 20/02/13. 